\title{
Cone beam computed tomographic survey of the mesiobuccal root canal anatomy in the maxillary first and second molar teeth of an Iranian population
}

\author{
Negar Khosravifard ${ }^{1}$, Zahra Dalili Kajan ${ }^{1}$, Homayoon Hasanpoor ${ }^{2}$
}

Correspondence: Dr. Zahra Dalili Kajan

Email: zahradalili@yahoo.com

\begin{abstract}
'Dental Sciences Research Center, Department of Maxillofacial Radiology, Faculty of Dentistry, Guilan University of Medical Sciences, Rasht, Iran, ${ }^{2}$ Faculty of Dentistry, Guilan University of Medical Sciences, Rasht, Iran
\end{abstract}

\section{ABSTRACT}

Objective: The objective of this study was to evaluate the mesiobuccal (MB) root canal(s) anatomy of the maxillary first and second molar teeth in an Iranian population. Materials and Methods: Cone beam computed tomography images of 280 teeth (145 maxillary first molars and 135 maxillary second molars) were analyzed to determine the number and types of the MB root canals. Chi-square test was used to examine the relationship between the number of MB canals and sex and Mann-Whitney analysis was used to define the relationship between the number of canals and the buccopalatal dimension of the MB roots. The level of interobservers' agreement was assessed with Kappa test. Results: Nearly $44.8 \%$ of the first molar and $18.5 \%$ of the second molar teeth had an additional MB canal (MB2). Types I and III of root canals had the highest frequencies. There was no significant relationship between the number of MB root canals of maxillary first and second molars and sex $(P=0.829)$. The relationship between the buccopalatal dimension of the MB roots and the number of root canals was shown to be statistically significant $(P=0.001)$. Conclusion: Presence of MB2 had a relatively high frequency, especially in the maxillary first molars. The mean buccopalatal dimension of the MB roots showed to be interrelated with the number of root canals.

Key words: Cone beam computed tomography, maxilla, root canal anatomy

\section{INTRODUCTION}

Knowledge of internal morphology of the root canal system is the main part of planning and performing endodontic treatments. ${ }^{[1]}$ The main purpose of endodontic treatments is to perform mechanical and chemical debridement of the whole length of the canal and fill it with biocompatible materials. ${ }^{[2]}$ On the other hand, inability to properly identify, debride, and fill all canals of a tooth is considered as the main

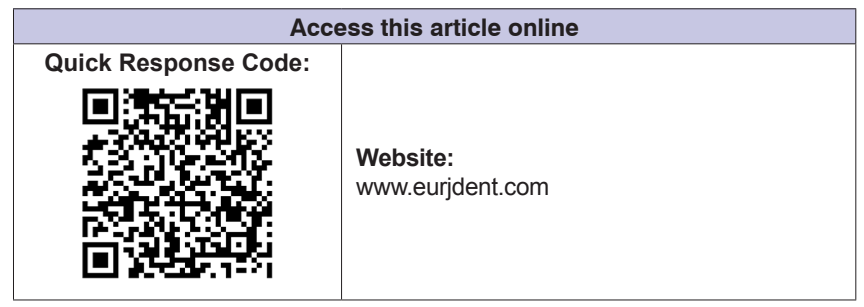

cause of endodontic treatment failure. ${ }^{[3]}$ Due to the more complex anatomy and common morphological variations, especially the presence of second mesiobuccal (MB2) canal and its various forms, attempts have been made in various studies to focus more on the $\mathrm{MB}$ root of maxillary molars. ${ }^{[2,4-12]}$ The high incidence of endodontic treatment failure in maxillary

This is an open access journal, and articles are distributed under the terms of the Creative Commons Attribution-NonCommercial-ShareAlike 4.0 License, which allows others to remix, tweak, and build upon the work non-commercially, as long as appropriate credit is given and the new creations are licensed under the identical terms.

For reprints contact: reprints@medknow.com

How to cite this article: Khosravifard N, Kajan ZD, Hasanpoor $\mathrm{H}$ Cone beam computed tomographic survey of the mesiobuccal root canal anatomy in the maxillary first and second molar teeth of an Iranian population. Eur J Dent 2018;12:422-7.

DOI: 10.4103/ejd.ejd_60_18 
first molars is probably due to the inability to find and fill the MB2 canal. Finding the location of MB2 is therefore the most important aspect of treatment of these canals. ${ }^{[13]}$ Several factors including the race and age as well as variations in the research methods are responsible for the widespread variations in the frequencies reported for MB2 in maxillary molars. ${ }^{[2]}$ Methods of root canal morphology evaluation include tooth clearing and staining, analog radiography, digital radiography, microcomputed tomography $(\mathrm{CT})$, cone beam CT (CBCT), CT, and modified tooth clearing and staining. ${ }^{[2]}$ Lack of anatomical superimpositions provides higher diagnostic accuracy for CBCT compared to digital and conventional periapical radiographies. ${ }^{[14]}$ In addition, in comparison with the conventional CT, CBCT has higher accuracy, higher resolution, less scan time, and low absorption dose. ${ }^{[15]}$ Multiple demographic studies have used CBCT for evaluation of the root shape and root canals in different teeth. ${ }^{[2,3,13,15,16]}$ Furthermore, several studies have been carried out on the root canal morphology of the Iranian population using CBCT images. ${ }^{[5,7,8,17,18]}$ Results of the mentioned researches are fairly inconsistent possibly due to the variations in the racial characteristics of different populations even in the same country. Hence, the present study was aimed at evaluating the $\mathrm{MB}$ root canal(s) anatomy in the maxillary first and second molar teeth of a northern Iranian population as well as determining the frequency of MB2 canals' association with the patients' sex and the buccopalatal dimension of the roots.

\section{MATERIALS AND METHODS}

In this cross-sectional study, CBCT images of 280 maxillary first and second molar teeth (145 first molars and 135 second molars) were used. The images belonged to patients who either attended the dental faculty of Rasht, Iran, or a private maxillofacial radiology center in Rasht, Iran, in the years 2016 and 2017. These images were previously acquired for different purposes including implant-site assessment or impacted tooth surgery. CBCT images were acquired by the same device ( New Tom, Verona, Italy) and with the following exposure parameters: voxel size: $0.2-0.24 \mathrm{~mm}$, field of view: $10 \mathrm{~cm} \times 10 \mathrm{~cm}$ for standard zoom mode and $22.5 \mathrm{~cm} \times 22.5 \mathrm{~cm}$ for full-zoom mode. The ethical approval code of this research was IR.GUMS.REC.1395.299 (registered by Guilan University of Medical Sciences). All of the images had to be of proper quality and include the upper jaw of the patients so that examination of the maxillary first and second molars would be possible.
Images of the maxillary first and second molars with severe caries extended to the cervical or intermediate portion of the root and also endodontically treated teeth in which the root canal treatment procedure had disrupted the original morphology of the root canal system were excluded from the study. Furthermore, first and second molar teeth with two roots were not included in the examination.

Axial and cross-sectional CBCT views with 1-mm slice thickness and 1-mm slice interval were evaluated by scrolling the images from the pulp chamber to the apex for each first and second molar tooth [Figures 1 and 2]. Two maxillofacial radiologists individually evaluated the CBCT images. Presence of MB2 canal within the MB roots of maxillary first and second molar teeth was assessed. Root canal configuration was determined according to the classification of Weine ${ }^{[19]}$ which describes the root canal types as follows: Type I - a root canal extending from the pulp chamber to the apex, Type II - two root canals that connect near the apex, Type III - two root canals leading to two distinct apical foramens, and Type IV - a root canal that is divided into two canals and ends at two distinct apical foramens. In addition to determining the number and shape of the MB root canals of the maxillary first and second molars, the relationship between the number of canals and patients' sex as well as the buccopalatal dimension of the MB roots at the level of cementoenamel junction where the canal orifice begins was evaluated. Chi-square test was used to examine the relationship between the number of MB canals and sex and Mann-Whitney analysis was used to define the relationship between the number of canals and the buccopalatal dimension of the MB

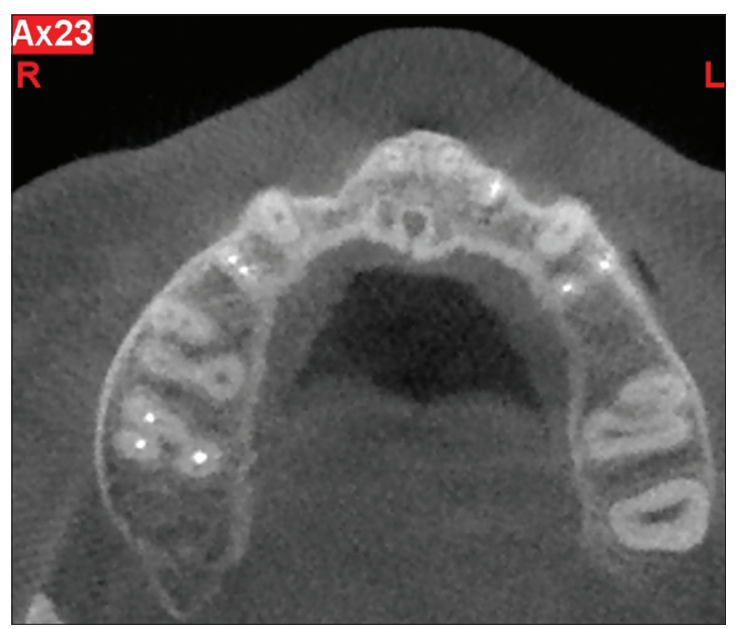

Figure 1: Axial cone beam computed tomography view shows two distinct canals in the mesiobuccal root of the maxillary right first molar tooth 


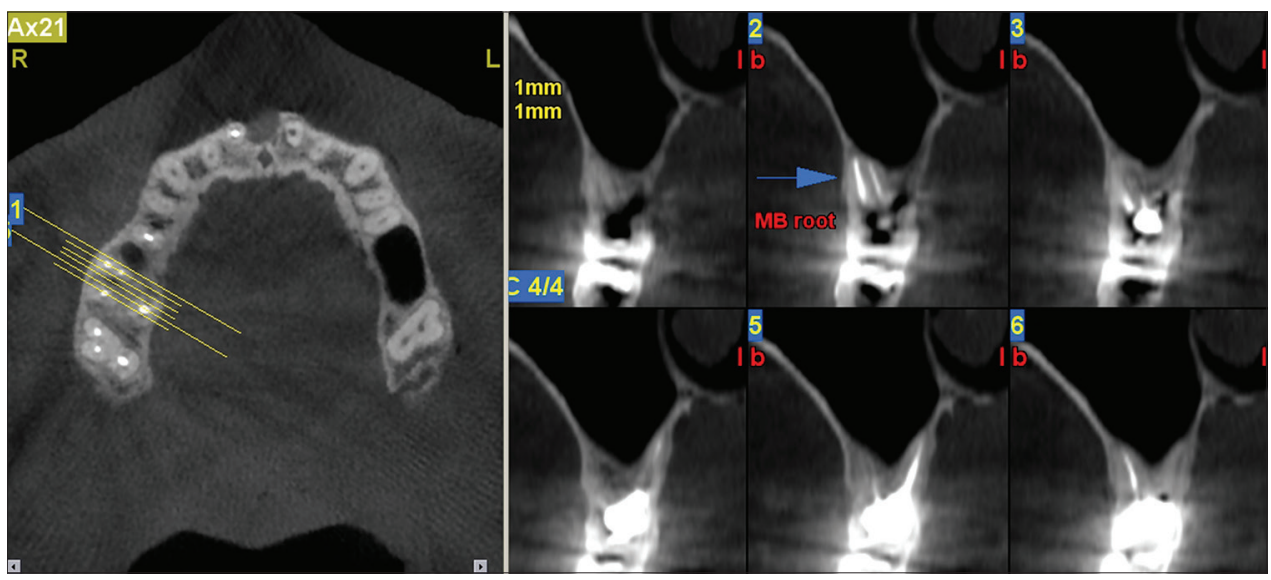

Figure 2: Axial and cross-sectional cone beam computed tomography views demonstrate two canals associated with the mesiobuccal root of an endodontically treated maxillary right first molar tooth

roots. Level of statistical significance was considered to be $<0.05$. The level of interobserver agreement was assessed with Kappa test.

\section{RESULTS}

In this study, a total of 280 teeth, including 145 maxillary first molars $(51.79 \%)$ and 135 maxillary second molars $(48.21 \%)$, were evaluated using CBCT images of an Iranian population.

Frequency of second mesiobuccal in the mesiobuccal roots of maxillary first and second molars

Nearly $44.8 \%$ of the maxillary first molars were shown to have MB2 and $55.2 \%$ had only one canal in the MB roots. In the maxillary second molars, it was revealed that $18.5 \%$ of the teeth had MB2 in the MB roots and $81.5 \%$ had a single canal in the mentioned roots. It should be noted that none of the samples were found to have more than two canals in their MB roots.

\section{Frequency of root canal types}

The highest and lowest frequencies of the canal types in the $\mathrm{MB}$ roots of the maxillary first molars were related to Type I (55.2\%) and Type IV (2.8\%), respectively. Similarly, in the maxillary second molars, Type I with the frequency of $81.5 \%$ and Type IV with the frequency of $0.7 \%$ had the highest and lowest frequencies, respectively [Table 1].

\section{Relationship between the number of mesiobuccal root canals and sex}

The CBCT images evaluated in the current study belonged to 182 women (65\%) and 98 men (35\%). Table 2 shows the number of MB root canals in the maxillary first and second molars according to the patients' sex. No significant relationships were obtained between the number of $\mathrm{MB}$ root canals and sex in both the first and second molar teeth.

Relationship between root dimensions and the number of canals in the mesiobuccal roots

According to the findings of Table 3, a significant relationship existed between the buccopalatal dimension of the MB roots measured at the onset of the orifice and the number of $\mathrm{MB}$ root canals in the maxillary first and second molars $(P<0.001)$ so that the mean buccopalatal dimension of the MB roots tended to be greater in the roots containing two canals.

\section{DISCUSSION}

The present study was mainly aimed at determination of the frequency of MB2 canals and their configuration types according to the classification of Weine ${ }^{[19]}$ in the first and second molar teeth of the maxilla in a northern Iranian population. Besides, assessment of any possible relationships between the presence of MB2 canals and the buccopalatal dimension of the MB roots as well as the patients' sex was performed. Using CBCT images, the frequency of MB2 canals was calculated to be $44.8 \%$ in the first molar and $18.5 \%$ in the second molar teeth. Regarding the configuration of canals based on the classification of Weine, ${ }^{[19]}$ the most encountered type of the MB canals was Type I. Possibility of the presence of two canals in the MB roots was higher in roots with greater buccopalatal dimensions. No sex predilection was found associated with the presence of MB2 in both the first and second maxillary molar teeth.

Successful nonsurgical root canal treatment requires mechanical and chemical debridement and three-dimensional filling of the root canals. ${ }^{[20]}$ 
Khosravifard, et al.: The mesiobuccal root canal anatomy of maxillary molars

\begin{tabular}{|c|c|c|c|c|c|}
\hline \multirow[t]{2}{*}{ Tooth } & \multicolumn{4}{|c|}{ MB canal type } & \multirow[t]{2}{*}{ Total $(n)$} \\
\hline & Type I, $\boldsymbol{n}(\%)$ & Type II, $\boldsymbol{n}(\%)$ & Type III, $\boldsymbol{n}(\%)$ & Type IV, $\boldsymbol{n}(\%)$ & \\
\hline Maxillary first molar & $80(55.2)$ & $17(11.7)$ & $44(30.3)$ & $4(2.8)$ & 145 \\
\hline Maxillary second molar & $110(81.5)$ & $7(5.2)$ & $17(12.6)$ & $1(0.7)$ & 135 \\
\hline
\end{tabular}

\begin{tabular}{|c|c|c|c|c|c|}
\hline \multirow[t]{2}{*}{ Tooth } & \multirow{2}{*}{$\begin{array}{l}\text { Number } \\
\text { of MB root } \\
\text { canals }\end{array}$} & \multicolumn{2}{|c|}{ Gender } & \multirow[t]{2}{*}{ Total } & \multirow[t]{2}{*}{$P^{*}$} \\
\hline & & $\begin{array}{l}\text { Male, } \\
n(\%) \\
\end{array}$ & $\begin{array}{c}\text { Female, } \\
n(\%)\end{array}$ & & \\
\hline \multirow{2}{*}{$\begin{array}{l}\text { Maxillary } \\
\text { first molar }\end{array}$} & 1 & $26(32.5)$ & $54(67.5)$ & 80 & 0.455 \\
\hline & 2 & 25 (38.5) & $40(61.5)$ & 65 & \\
\hline \multirow{2}{*}{$\begin{array}{l}\text { Maxillary } \\
\text { second molar }\end{array}$} & 1 & $41(37.3)$ & $69(62.7)$ & 110 & 0.209 \\
\hline & 2 & $6(24)$ & $19(76)$ & 88 & \\
\hline
\end{tabular}

Table 3: Frequency distribution of number of
mesiobuccal root canals according to root dimensions

${ }^{*}$ Mann-Whitney. SD: Standard deviation, MB: Mesiobuccal

Due to the more complex anatomy, particularly the presence of a MB2, various studies and researches have focused on the $\mathrm{MB}$ roots of maxillary molars by different methods. ${ }^{[2-6,18]}$ The ideal method should have definite criteria including accuracy, simplicity, and applicability for in vivo conditions. ${ }^{[3]}$ Today, CBCT has been used as a comparative method for studying the roots and root canal anatomy of human teeth for in vivo environments and numerous studies have benefited from this imaging technique for evaluation of the root form and root canal anatomy in different teeth. ${ }^{[2-4,6,7]}$ It has been shown that in order to find the MB2 canal of the maxillary molar teeth, CBCT is a reliable method in comparison with the gold standard of tooth sectioning. ${ }^{[13]}$

Results of the present study indicated that $44.8 \%$ of the first molars and $18.5 \%$ of the second molars had MB2 canals. Furthermore, with respect to the root canal type in accordance to the classification of Weine, ${ }^{[19]}$ the obtained frequencies in descending order were as follows: Type I, Type III, Type II, and Type IV. Rouhani et al. ${ }^{[7]}$ investigated the root canal morphology of a number of extracted maxillary first and second molar teeth of an Iranian population via $\mathrm{CBCT}$ imaging. According to the classification of
Vertucci, they found out that the most frequent canal type in all the three roots of both the first and second molar teeth was Type I. On the contrary, evaluation of the CBCT images of another Iranian population by Khademi et al. ${ }^{[8]}$ revealed that in both the first and second molar teeth of the maxilla, Type II of the Vertucci classification had the highest frequency in the MB roots. They also found the frequency of MB2 canals to be $70.2 \%$ in the first molars and $43.4 \%$ in the second molars. Another investigation on the Iranian population performed by Naseri et al. ${ }^{[17]}$ came upon a frequency as great as $86.6 \%$ for MB2 canals in the maxillary first molar teeth. The variations that are encountered in both the frequency and type of the MB2 canals in the mentioned studies are best explained by the racial characteristics of the populations selected from the different regions of Iran, worthy to mention that none were previously performed on the northern population as our research did.

In the study of Baratto Filho et al., ${ }^{[1]}$ which was aimed at investigating the internal morphology of the maxillary first molars, 54 teeth were examined using CBCT. It was shown that $37.05 \%$ of three-rooted teeth had four canals. In $90.90 \%$ of the teeth with four root canals, only one apical foramen was seen which could be considered analogous to the Type I of Weine's classification. ${ }^{[19]}$ Results of this study were quite close to ours in terms of frequency and type of MB2 canals in the first molar teeth of the maxilla.

Lin et al. ${ }^{[4]}$ estimated the frequency of MB2 canals to be $56 \%$ in the first molar and $7.7 \%$ in the second molar teeth of a Taiwanese population using CBCT images. Another study performed by Celikten et al. ${ }^{[6]}$ on the CBCT images of a Turkish population revealed that the frequency of MB2 canals was $50.6 \%$ and $22.8 \%$ in the maxillary first and second molar teeth, respectively, which is approximately close to the results of our study. They also reported that on the basis of Vertucci classification, Type I in the first molars and Type II in the second molars were the most frequent varieties.

Shetty et al. ${ }^{[10]}$ evaluated the CBCT images of endodontically treated maxillary first and second molar teeth in an Indian population. They reported 
that the frequency of MB2 canals was $86.63 \%$ in the first molar and $29.4 \%$ in the second molar teeth. Based on their findings, $77.19 \%$ of the MB2 canals of the first molars and $90 \%$ of them in the second molars had been missed during the root canal treatment procedure and subsequently $72.7 \%$ of the first molars and $88.8 \%$ of the second molars showed to have periapical radiolucencies.

Zheng et al. ${ }^{[15]}$ evaluated the root form and root canal anatomy of maxillary first molar teeth using CBCT images in a Chinese population. They reported that the frequency of MB2 canals was $52.24 \%$. The frequency of MB2 canals in this study was highly consistent with the findings of the studies performed on a selected population of Greece $(53.4 \%)^{[12]}$ and the Asian populations of Thailand $(65 \%)^{[21]}$ and Japan $\left.(58 \%)\right)^{[22]}$ but obviously lower than the frequencies reported for a Turkish $(93.5 \%)^{[23]}$ and a Pakistani $(70.6 \%)$ population. ${ }^{[11]}$

Silva et al. ${ }^{[24]}$ reported that the frequency of MB2 canals was $42.63 \%$ in the maxillary first molar and $34.32 \%$ in the maxillary second molar teeth using CBCT images in a Brazilian population. Furthermore, regarding the root canal types based on the Weine's classification, they claimed that Type I and Type III were the most frequently detected varieties, a finding in complete agreement with the results of our study as well as the study of Neelakantan et al. that investigated the root canal morphology of maxillary first and second molar teeth in an Indian population via CBCT images. ${ }^{[3]}$

The wide variations that are observed among different studies on the frequency and types of the MB2 canals in the maxillary molar teeth could be explained by the differences in ethnicity, sample size, study design (including exclusion/inclusion criteria and whether it is conducted clinically or experimentally), and method of root canal detection and evaluation.

In the current study, no significant relationships were found between the patients' sex and the number of MB2 canals in the maxillary first and second molar teeth $(P=0.829)$, a finding that is consistent with the study results of Celikten et al. ${ }^{[6]}$ Gomes Alves et al., ${ }^{\left[{ }^{[j]}\right.}$ and Zheng et al., ${ }^{[15]}$ however in contradiction with the results of the study of Sert and Bayirli. ${ }^{[23]}$ On the other hand, Kim et al..$^{[25]}$ concluded that although no significant relationship is seen between gender and presence of MB2 in the maxillary second molar teeth, it seems that men have higher rates of MB2 canals in their maxillary first molars.
Mohammadzadeh Akhlaghi et al. ${ }^{[18]}$ assessed the thickness of the MB roots in the maxillary first molar teeth and arrived at the conclusion that in the roots with a single canal, the mesial and distal aspects of the root have the least thickness and in the roots containing two root canals, the thinnest parts are the distobuccal and the distopalatal sides. According to the findings of the present study, a significant relationship was found between the presence of MB2 canals in the maxillary first and second molars and the buccopalatal dimension of the $\mathrm{MB}$ roots measured at the orifice level. The mean buccopalatal dimension of the $\mathrm{MB}$ roots at the onset of the canal orifices tends to be greater in roots having two root canals.

\section{CONCLUSION}

In the present study, MB2 canals did not show a very high frequency in the maxillary first and second molar teeth, which is highly associated with the racial features of the population. With respect to the canal types according to the classification developed by Weine, Type I and Type III had the highest frequencies. There was no relationship between sex and the frequency of MB2 canals; on the contrary, the mean buccopalatal dimension of the MB roots showed to be interrelated with the number of root canals.

\section{Financial support and sponsorship \\ Nil.}

\section{Conflicts of interest}

There are no conflicts of interest.

\section{REFERENCES}

1. Baratto Filho F, Zaitter S, Haragushiku GA, de Campos EA, Abuabara A, Correr GM, et al. Analysis of the internal anatomy of maxillary first molars by using different methods. J Endod 2009;35:337-42.

2. Lee JH, Kim KD, Lee JK, Park W, Jeong JS, Lee Y, et al. Mesiobuccal root canal anatomy of korean maxillary first and second molars by cone-beam computed tomography. Oral Surg Oral Med Oral Pathol Oral Radiol Endod 2011;111:785-91.

3. Neelakantan P, Subbarao C, Ahuja R, Subbarao CV, Gutmann JL. Cone-beam computed tomography study of root and canal morphology of maxillary first and second molars in an Indian population. J Endod 2010;36:1622-7.

4. Lin $\mathrm{YH}, \mathrm{Lin} \mathrm{HN}, \mathrm{Chen} \mathrm{CC}$, Chen MS. Evaluation of the root and canal systems of maxillary molars in Taiwanese patients: A cone beam computed tomography study. Biomed J 2017;40:232-8.

5. Rezaeian M, Rouhani Tonekaboni M, Iranmanesh F. Evaluating the root canal morphology of permanent maxillary first molars in Iranian population. Iran Endod J 2018;13:78-82.

6. Celikten B, Tufenkci P, Aksoy U, Kalender A, Kermeoglu F, Dabaj P, et al. Cone beam CT evaluation of mandibular molar root canal morphology in a Turkish Cypriot population. Clin Oral Investig 2016;20:2221-6.

7. Rouhani A, Bagherpour A, Akbari M, Azizi M, Nejat A, Naghavi N, et al. Cone-beam computed tomography evaluation of maxillary first 
and second molars in Iranian population: A morphological study. Iran Endod J 2014;9:190-4.

8. Khademi A, Zamani Naser A, Bahreinian Z, Mehdizadeh M, Najarian M, Khazaei S, et al. Root morphology and canal configuration of first and second maxillary molars in a selected Iranian population: A cone-beam computed tomography evaluation. Iran Endod J 2017;12:288-92.

9. Gomes Alves CR, Martins Marques M, Stella Moreira M, Harumi Miyagi de Cara SP, Silveira Bueno CE, Lascala CÂ, et al. Second mesiobuccal root canal of maxillary first molars in a Brazilian population in high-resolution cone-beam computed tomography. Iran Endod J 2018;13:71-7.

10. Shetty H, SontakkeS, Karjodkar F, Gupta P, Mandwe A, Banga KS, et al. A cone beam computed tomography (CBCT) evaluation of MB2 canals in endodontically treated permanent maxillary molars. A retrospective study in Indian population. J Clin Exp Dent 2017;9:e51-5.

11. Alrahabi M, Sohail Zafar M. Evaluation of root canal morphology of maxillary molars using cone beam computed tomography. Pak J Med Sci 2015;31:426-30.

12. Nikoloudaki GE, Kontogiannis TG, Kerezoudis NP. Evaluation of the root and canal morphology of maxillary permanent molars and the incidence of the second mesiobuccal root canal in Greek population using cone-beam computed tomography. Open Dent J 2015;9:267-72.

13. Blattner TC, George N, Lee CC, Kumar V, Yelton CD. Efficacy of cone-beam computed tomography as a modality to accurately identify the presence of second mesiobuccal canals in maxillary first and second molars: A pilot study. J Endod 2010;36:867-70.

14. Yang H, Tian C, Li G, Yang L, Han X, Wang Y, et al. A cone-beam computed tomography study of the root canal morphology of mandibular first premolars and the location of root canal orifices and apical foramina in a Chinese subpopulation. J Endod 2013;39:435-8.

15. Zheng QH, Wang Y, Zhou XD, Wang Q, Zheng GN, Huang DM, et al. A cone-beam computed tomography study of maxillary first permanent molar root and canal morphology in a Chinese population. J Endod 2010;36:1480-4.

16. Pauwels R, Araki K, Siewerdsen JH, Thongvigitmanee SS. Technical aspects of dental CBCT: State of the art. Dentomaxillofac Radiol 2015;44:20140224.

17. Naseri M, Safi Y, Akbarzadeh Baghban A, Khayat A, Eftekhar L. Survey of anatomy and root canal morphology of maxillary first molars regarding age and gender in an Iranian population using cone-beam computed tomography. Iran Endod J 2016;11:298-303.

18. Mohammadzadeh Akhlaghi N, Ravandoust $Y$, Najafi M, Dadresanfar B. An in vitro study of mesiobuccal root thickness of maxillary first molars. Iran Endod J 2012;7:31-5.

19. Weine FS. Endodontic Therapy. $5^{\text {th }}$ ed. St. Louis: Mosby; 1996.

20. Berman LH, Hargreaves KM, Cohen SR. Cohen's Pathways of the Pulp Expert Consult. 10 $0^{\text {th }}$ ed. St. Louis: Mosby; 2011. p. 138.

21. Gulabivala K, Opasanon A, Ng YL, Alavi A. Root and canal morphology of Thai mandibular molars. Int Endod J 2002;35:56-62.

22. Neelakantan P, Subbarao C, Subbarao CV. Comparative evaluation of modified canal staining and clearing technique, cone-beam computed tomography, peripheral quantitative computed tomography, spiral computed tomography, and plain and contrast medium-enhanced digital radiography in studying root canal morphology. J Endod 2010;36:1547-51.

23. Sert S, Bayirli GS. Evaluation of the root canal configurations of the mandibular and maxillary permanent teeth by gender in the Turkish population. J Endod 2004;30:391-8.

24. Silva EJ, Nejaim Y, Silva AI, Haiter-Neto F, Zaia AA, Cohenca N, et al. Evaluation of root canal configuration of maxillary molars in a Brazilian population using cone-beam computed tomographic imaging: An in vivo study. J Endod 2014;40:173-6.

25. Kim Y, Lee SJ, Woo J. Morphology of maxillary first and second molars analyzed by cone-beam computed tomography in a Korean population: Variations in the number of roots and canals and the incidence of fusion. J Endod 2012;38:1063-8. 